\title{
Comparison of Sensitivity of HbA1c with Fasting Blood Glucose for Diagnosing Prediabetes in Chinese Americans
}

\author{
Andrew Wang ${ }^{1}$, Qiuhu Shi ${ }^{2}$, John $\mathrm{CH}^{1}$ and Jaime Uribarri ${ }^{1 *}$ \\ ${ }^{1}$ Department of Medicine, Icahn School of Medicine at Mount Sinai, USA \\ ${ }^{2}$ New York Medical College, USA
}

Submission: November 01, 2017; Published: November 27, 2017

*Corresponding author: Jaime Uribarri, Division of Nephrology, Department of Medicine, Icahn School of Medicine at Mount Sinai, One Gustave Levy Pl, Box 1147, New York- 10029, USA, Tel: 212-241-1887; Fax: 646-537-8627; Email: Jaime.uribarri@mssm.edu

\section{Abstract}

Background: In 2010 The American Diabetes Association (ADA) recommended HbA1c as an alternative to fasting blood glucose (FBG) as a screening test when diagnosing prediabetes, but little is known about sensitivity of HbA1c and fasting glucose for diagnosing prediabetes in Chinese Americans in primary care setting.

Methods: Data from a primary care clinic were analyzed to determine the sensitivity of elevated HbA1c (5.7\%-6.4\%) and FBG (100-125mg/ dc) for diagnosing prediabetes. Prediabetes was diagnosed as per ADA suggested criteria: HbA1c between $5.7 \%-6.4 \%$ or fasting glucose between $100-125 \mathrm{mg} / \mathrm{dl}$ (or OGTT if done). A total of 1480 patients with both FBG and HbA1c measured at the same time were included from 11/2016 to $07 / 2017$.

Results: The prevalence of total prediabetes for isolated $\mathrm{HbA1c}$, isolated FBG, and their overlapping was $47.1 \%, 3.2 \%$, and $14.7 \%$ respectively. Using abnormal FBG as the reference standard, the sensitivity for diagnosing prediabetes by HbA1c criteria is $82.3 \%$.

Conclusions: In this population of Chinese Americans, HbA1c has much higher sensitivity to diagnose prediabetes, in contrast to the sensitivity described in the literature for non-Hispanic whites, non-Hispanic blacks and Hispanic Americans. The prevalence of prediabetes by HbA1c alone is much higher in this group than that described in the literature by FBG alone in Chinese Americans.

Keywords: Chinese americans; Pre-diabetes; Insulin sensitivity; Diabetes

\section{Introduction}

Prediabetes is a chronic condition, associated with increased risk of diabetes-related complications, such as heart disease, stroke, and nephropathy etc. The definition of prediabetes by the American Diabetes Association (ADA) is HbA1c between 5.7\%$6.4 \%$ (or fasting glucose between $100-125 \mathrm{mg} / \mathrm{dl}$ or OGTT if done) [1]. Prediabetes is becoming more and more common in the US. More than one third of American adults, around 84.1million, had prediabetes in 2015, based on their HbA1c or FG, and $90 \%$ of them do not know it [2]. Nearly half (48.3\%) of adults aged 65 or above had prediabetes [2]. In addition, about $25 \%$ of individuals with prediabetes will develop diabetes over the next 3-5 years. With longer observation, most individuals with prediabetes eventually develop diabetes, which in turn causes major morbidity and mortality [3]. Individuals with additional risk factors, including overweight, family history, elderly, are more likely to develop diabetes. Diabetes places a tremendous demand on the economical resources of the US with about $20 \%$ of the nation's health care dollars going to treating people with diabetes [4]. In order to save the government billions of dollars in medical care costs associated with prediabetes progression to diabetes, the Centers for Medicare \& Medicaid Services (CMS), in partnership with CDC, developed the Medicare Diabetes Prevention Program, which will be offered at no cost to participants (but with reimbursement to medical providers) beginning Jan 1, 2018. The program focuses on overweight Medicare beneficiary with prediabetes through weight loss and education [5]. A study by Yeh [6] etc specifically focusing on Chinese Americans with prediabetes showed lifestyle 
intervention significantly decreased diabetes progression by improving HbA1c.

In 2015, Chinese Americans were the largest fastest growing ethnic group among Asian Americans, which in turn were the nation's fastest-growing ethnic group, with a growth rate over 4 times that of the total US in 2012 [7,8]. The diabetes prevalence in Chinese Americans was 4.3\% in 2013-2015 [2] with ranges from $2.2-28.0 \%$ [7]. The age-adjusted prevalence of prediabetes was 35.7\% in Asian American in 2011-2014 (no specific numbers for Chinese Americans), which was similar among in non-Hispanic whites, non-Hispanic blacks and Hispanic Americans [2]. In the current study, we have used data from a primary care clinic in New York City to compare the sensitivity, prevalence of HbA1c and FBG for diagnosing prediabetes in a group of Chinese Americans.

\section{Subjects/Methods}

Clinical and laboratory data from individuals older than 18 years of age, of any gender, and attending a primary care facility for yearly physical checkup or regular follow up were incorporated into a de-identified database from November, 2016 to July, 2017 and used for statistical analyses. Diabetes was diagnosed by fasting blood glucose $\geq 126 \mathrm{mg} / \mathrm{dl}$, HbA1C $\geq 6.5 \%$ or by history. Also, individuals with certain conditions affecting HbA1c measurement such as anemia (defined by $\mathrm{Hb}<$ normal lower cut off for each individual laboratory), hemoglobinopathy, hemolysis, recent blood loss/transfusion, erythropoietin therapy, pregnancy and chronic liver disease were excluded.

The definition of prediabetes was the one used by the American Diabetes Association (ADA): HbA1c between 5.7\%$6.4 \%$ or fasting glucose between $100-125 \mathrm{mg} / \mathrm{dl}$ (or OGTT if done) [1]. The study was done in a primary care clinic in Flushing, New York City. The majority of the patients in this clinic are Chinese (more than 95\%). Chinese are defined as individuals with a Chinese last name such as Wang, Li, etc, who also can speak Chinese. Only data in those subjects who had both HbA1c and FBG levels determined at the same time were included $(\mathrm{n}=1480)$.

Since all analyses were performed using a database with no identifiers and previously collected, the Icahn School of Medicine at Mount Sinai Program for the Protection of Human subjects (IRB) was consulted and decided that the study did not require IRB approval or signed participants' consent.

Majority of fasting blood samples (fasting at least 8 hours) were drawn at the clinic and the specimen picked up at evening or nighttime by an employee from Quest Diagnostics (New York, USA). The remainder of fasting blood samples was drawn at local (Flushing, New York City) branches from Quest Diagnostics, Bio Reference Laboatories (Elmwood, NJ) or Lab Corp (Burlington, $\mathrm{NC}$ ). HbA1c, FBG as well as all other biochemical parameters were all measured at Quest Diagnostics, Bio Reference or Lab Corp central laboratories. Demographic data (age, gender) and vital signs (systolic and diastolic blood pressure and weight and height) were all documented during the subjects' clinic visit.

\section{Statistical analysis}

Descriptive statistics number and percentage are used to summarize categorical and biochemical variables, and mean and standard deviation for continuous variables. Prevalence of isolated $\mathrm{HbA} 1 \mathrm{c}$ and isolated FBG were calculated. Every subject had both HbA1c and FBG test values. The prevalence of prediabetes was calculated based on ADA suggested criteria described above. Two-test agreement was tested by Chi-Square test. The abnormal FBG subjects were used as the reference. The sensitivity of HbA1c was defined by the number of abnormal HbA1c among the abnormal FBG subjects. The sensitivity corresponding various cut values of HbA1c was calculated. All analyses were performed using SAS version 9.4.

\section{Results}

Table 1: Characteristics of participants by $\mathrm{HbA} 1 \mathrm{c}$ and fasting blood glucose.

\begin{tabular}{|c|c|c|c|c|c|}
\hline \multirow[t]{2}{*}{$\mathbf{N}$} & \multicolumn{2}{|c|}{ HbA1c $<5.7 \%$} & \multicolumn{2}{|c|}{ HbA1c $>5.7 \%$} & \multirow[b]{2}{*}{ Total } \\
\hline & $\mathrm{FBG}<100 \mathrm{mg} / \mathrm{dl}$ & FBG $>100 \mathrm{mg} / \mathrm{dl}$ & FBG $<100 \mathrm{mg} / \mathrm{dl}$ & FBG $>100 \mathrm{mg} / \mathrm{dl}$ & \\
\hline & 517 & 47 & 697 & 219 & 1480 \\
\hline $\mathrm{HbA1c}(\%)$ & $5.4 \pm 0.2$ & $5.5 \pm 0.1$ & $5.9 \pm 0.2$ & $6.0 \pm 0.2$ & $5.7 \pm 0.3$ \\
\hline $\mathrm{FBG}(\mathrm{mg} / \mathrm{dl})$ & $85.5 \pm 7.5$ & $105.4 \pm 5.9$ & $88.6 \pm 6.7$ & $106.1 \pm 5.4$ & $90.6 \pm 10.0$ \\
\hline Age(years) & $47.7 \pm 13.9$ & $54.7 \pm 12.3$ & $58.0 \pm 11.8$ & $58.9 \pm 10.7$ & $54.4 \pm 13.4$ \\
\hline Female(\%) & 55.1 & 31.9 & 58.1 & 45.7 & 54.4 \\
\hline Current smoker(\%) & 10.3 & 21.3 & 9.6 & 9.1 & 10.2 \\
\hline Systolic blood pressure (mmHg) & $119.3 \pm 12.9$ & $125.2 \pm 14.5$ & $123.9 \pm 10.7$ & $127.7 \pm 12.2$ & $122.9 \pm 12.2$ \\
\hline Diastolic blood pressure (mmHg) & $75.2 \pm 7.9$ & $78.4 \pm 9.0$ & $77.5 \pm 7.2$ & $79.7 \pm 7.3$ & $77.1 \pm 7.7$ \\
\hline
\end{tabular}




\section{Current Research in Diabetes \& Obesity Journal}

\begin{tabular}{|c|c|c|c|c|c|}
\hline Hypertension(\%) & 23.1 & 34.0 & 43.2 & 47.0 & 36.4 \\
\hline BMI(kg/m $\left.{ }^{2}\right)$ & $23.8 \pm 3.2$ & $24.2 \pm 3.4$ & $24.7 \pm 3.1$ & $25.9 \pm 3.2$ & $24.6 \pm 3.2$ \\
\hline Total cholesterol (mg/dl) & $184.8 \pm 30.6$ & $182.1 \pm 32.8$ & $194.0 \pm 34.3$ & $195.8 \pm 38.0$ & $190.6 \pm 33.9$ \\
\hline HDL cholesterol(mg/dl) & $57.4 \pm 14.8$ & $56.9 \pm 15.3$ & $56.4 \pm 15.2$ & $53.9 \pm 13.9$ & $56.4 \pm 14.9$ \\
\hline LDL cholesterol(mg/dl) & $104.5 \pm 27.9$ & $95.8 \pm 24.8$ & $111.1 \pm 30.8$ & $115.2 \pm 33.5$ & $108.9 \pm 30.4$ \\
\hline Triglycerides(mg/dl) & $118.0 \pm 75.9$ & $135.8 \pm 74.6$ & $136.0 \pm 93.4$ & $136.6 \pm 78.4$ & $129.8 \pm 85.3$ \\
\hline eGFR<60 ml/min per 1.73m (\%) & 1.6 & 2.1 & 2.7 & 2.7 & 2.3 \\
\hline Hemoglobin(g/dl) & $14.0 \pm 1.3$ & $14.4 \pm 1.2$ & $13.8 \pm 1.2$ & $14.2 \pm 1.2$ & $14.0 \pm 1.2$ \\
\hline Serum Albumin(g/dl) & $4.5 \pm 0.3$ & $4.4 \pm 0.3$ & $4.4 \pm 0.2$ & $4.5 \pm 0.3$ & $4.4 \pm 0.3$ \\
\hline Aspartate aminotransferase (units/l) & $20.6 \pm 9.6$ & $24.4 \pm 14.1$ & $21.9 \pm 7.1$ & $22.3 \pm 8.1$ & $21.6 \pm 8.5$ \\
\hline Alanine aminotransferase (units/l) & $20.2 \pm 15.3$ & $21.6 \pm 10.3$ & $21.6 \pm 14.1$ & $23.4 \pm 14.9$ & $21.4 \pm 14.6$ \\
\hline
\end{tabular}

FBG = Fasting Blood Glucose; eGFR = Estimated Glomerular Filtration Rate by Equation

Mean+SD was used to summarize continuous variables and percentage for categorical variables.

Main characteristics of the study population are described (estimated glomerular filtration rate greater than $60 \mathrm{ml} / \mathrm{min}$ per in Table 1. There were 1480 subjects included in the analyses $1.73 \mathrm{~m} 2$ in $97.7 \%$ ). Serum albumin, hemoglobin, liver function with an average age of 54.4 years and about equally distributed tests and lipid levels were within normal limits. There were $10 \%$ between men and women. Most of them had normal renal who were current smokers.

Table 2: Distribution of adults without diagnosed diabetes by the cross-classification of $\mathrm{HbA} 1 \mathrm{c}$ and fasting blood glucose using different $\mathrm{HbA} 1 \mathrm{c}$ cut points.

\begin{tabular}{|c|c|c|c|c|}
\hline \multirow{2}{*}{ HbA1c Cut Point } & \multicolumn{2}{|c|}{$<$ HbA1c Cut Point } & \multicolumn{2}{|c|}{$>$ HbA1c Cut Point } \\
\hline & $\mathrm{FBG}<100 \mathrm{mg} / \mathrm{dl}$ & $\mathrm{FBG}>100 \mathrm{mg} / \mathrm{dl}$ & FBG $<100 \mathrm{mg} / \mathrm{dl}$ & FBG $>100 \mathrm{mg} / \mathrm{dl}$ \\
\hline $5.30 \%$ & $5.7(4.6,7.0)$ & $0.2(0.0,0.6)$ & $76.4(74.1,78.5)$ & $17.8(15.9,19.8)$ \\
\hline $5.40 \%$ & $10.9(9.4,12.6)$ & $0.7(0.4,1.3)$ & $71.1(68.7,73.4)$ & $17.2(15.3,19.3)$ \\
\hline $5.50 \%$ & $17.4(15.5,19.5)$ & $1.4(0.8,2.1)$ & $64.6(62.1,67.0)$ & $16.6(14.8,18.6)$ \\
\hline $5.60 \%$ & $26.4(24.1,28.7)$ & $1.9(1.3,2.7)$ & $55.7(53.1,58.2)$ & $16.1(14.2,18.1)$ \\
\hline $5.70 \%$ & $34.9(32.5,37.4)$ & $3.2(2.3,4.2)$ & $47.1(44.5,49.7)$ & $14.8(13.0,16.7)$ \\
\hline $5.80 \%$ & $46.1(43.5,48.7)$ & $5.3(4.2,6.5)$ & $35.9(33.5,38.5)$ & $12.7(11.0,14.5)$ \\
\hline $5.90 \%$ & $55.3(52.7,57.8)$ & $7.6(6.3,9.0)$ & $26.8(24.5,29.1)$ & $10.4(8.9,12.1)$ \\
\hline $6.00 \%$ & $64.1(61.5,66.5)$ & $9.7(8.2,11.3)$ & $18.0(16.0,20.0)$ & $8.3(7.0,9.8)$ \\
\hline $6.10 \%$ & $70.8(68.4,73.1)$ & $11.8(10.2,13.5)$ & $11.2(9.7,12.9)$ & $6.2(5.0,7.6)$ \\
\hline $6.20 \%$ & $76.4(74.2,78.6)$ & $14.3(12.6,16.2)$ & $5.6(4.5,6.9)$ & $3.6(2.8,4.7)$ \\
\hline $6.30 \%$ & $79.7(77.5,81.7)$ & $16.1(14.2,18.1)$ & $2.4(1.7,3.3)$ & $1.9(1.3,2.7)$ \\
\hline $6.40 \%$ & $81.1(79.1,83.1)$ & $17.4(15.5,19.5)$ & $0.9(0.5,1.5)$ & $0.5(0.2,1.1)$ \\
\hline
\end{tabular}

The prevalence of total prediabetes for isolated $\mathrm{HbA1c}$, isolated FBG, and their overlapping was $47.1 \%, 3.2 \%$, and $14.7 \%$ respectively (Table 2$)$. The use of FBG $(100-125 \mathrm{mg} /$ dl) for diagnosis of prediabetes failed to identify $72.4 \%$ of the patients with abnormal HbA1c (5.7-6.4), while the use of HbA1c for diagnosis of prediabetes only failed to identify $5 \%$ of the patients with abnormal FBG (100-125mg/dl).

The sensitivity for diagnosing prediabetes by HbA1c criteria was $82.3 \%$, using FBG as the reference standard (Table 3). 


\section{Current Research in Diabetes \& Obesity Journal}

Table 3: Sensitivity, specificity, positive predictive value and negative predictive value according to different $\mathrm{HbA} 1 \mathrm{c}$ cut points.

\begin{tabular}{|c|c|c|c|c|}
\hline \multicolumn{5}{|c|}{ Test Characteristics (\%) } \\
\hline HbA1c cut point & Sensitivity & Specificity & Positive predictive value & Negative predictive value \\
\hline $5.30 \%$ & 98.9 & 6.9 & 18.9 & 96.6 \\
\hline $5.40 \%$ & 95.9 & 13.3 & 19.5 & 93.6 \\
\hline $5.50 \%$ & 92.5 & 21.3 & 20.5 & 92.8 \\
\hline $5.60 \%$ & 89.5 & 32.1 & 22.4 & 93.3 \\
\hline $5.70 \%$ & 82.3 & 42.6 & 23.9 & 89.1 \\
\hline $5.80 \%$ & 70.9 & 56.2 & 28 & 89.7 \\
\hline $5.90 \%$ & 57.9 & 67.4 & 31.6 & 86.9 \\
\hline $6.00 \%$ & 46.2 & 78.1 & 35.7 & 85.8 \\
\hline $6.10 \%$ & 34.6 & 86.3 & 39.4 & 84.2 \\
\hline $6.20 \%$ & 20.3 & 93.2 & 44.4 & 83.2 \\
\hline $6.30 \%$ & 10.5 & 97.1 & 38.1 & 8.3 \\
\hline $6.40 \%$ & 3 & 98.9 & & 8 \\
\hline
\end{tabular}

Subjects who had abnormal FBG based on ADA suggestion criteria were used as case and normal FBG as control. Given a cut point, sensitivity, specificity, positive predictive value and negative predictive value were calculated based on these cases and controls defined by FBG.

\section{Discussion}

Since ADA recommended $\mathrm{HbA1c}$ as a screening test for prediabetes in 2010, HbA1c has become a preferred test over fasting glucose in the primary care setting due to its convenience, less day-to-day perturbation during stress and illness, and greater pre-analytical stability [1]. In our study population, made up by Chinese Americans attending a primary care clinic in Flushing, New York City, we found that the sensitivity of HbA1c criteria to diagnose prediabetes, using FBG as the reference standard was $82.3 \%$, a value which is much higher than that reported in majority of Americans (27.0\%). This sensitivity is also in contrast with published findings in non-Hispanic whites, non-Hispanic blacks and Hispanic Americans. In the latter populations, Mann et al showed that overall sensitivity of HbA1c criteria was 27\% using data from NHANES 1999-2006 [9].

The prevalence of prediabetes defined using $\mathrm{HbA1c}$ alone in our study population was much higher than that using FBG alone (47.1\% vs $3.2 \%$ ), also quite in contrast with findings in nonHispanic whites, non-Hispanic blacks, and Hispanic Americans. Guo et al. [10] using data from NHANES 2005-2010 showed the overall prevalence of prediabetes by $\mathrm{HbA1}$ c alone being much lower than that by FBG alone in non-Hispanic whites $(23.8 \%$ vs $45.3 \%$ ) and Mexican American (25.8\% versus $45.7 \%$ ), but almost equal in non-Hispanic blacks (37.4\% versus $38 \%$ ). Mann et al. [9]. using data from NHANES 1999-2006 also showed that overall prevalence of prediabetes was much lower in these ethnic groups using $\mathrm{HbA} 1 \mathrm{c}$ alone compared to FBG alone $16.5 \%$ versus $30.5 \%$ ).
The significant difference in sensitivity of HbA1c in our study group could be due to genetic and/or environmental factors. Asian Americans had higher HbA1c levels than whites (mean $5.96 \%$ versus $5.80 \%$ ) [11]. Racial difference in hemoglobin glycation or red cell survival might account for the difference in HbA1c level independent of glycemia [11,12]. Chen at all found Chinese and other east Asians have multiple non-glycemic genotypes in red cells, which affects hemoglobin de-glycation, causing $1.76 \%$ of the variance in HbA1c [13]. The relatively high percentage of smoking in Chinese Americans (both active and passive) may explain in part HbA1c by affecting hemoglobin glycation independently of glycemia [12]. Another factor to consider in our study was the delay in analysis of the blood samples taken to a national laboratory; this would affect FBG more than HbA1c due to the effect of glycolysis.

Reasons for the much higher prediabetes prevalence in this study by HbA1c versus FBG are probably multi factorial. Participants were from a primary care setting and therefore more likely to be health conscious but sicker than general population. Moreover, mean BMI was higher in this study than the ADA recommended screening BMI of 23 for Asian Americans [2], which might detect more prediabetes. Lear et al. [14] found more abdominal fat in Chinese Canadians than Whites. Visceral fat may have an independent increased risk of getting diabetes [15]. White rice and refined grain makes up large portion of daily energy intake in Chinese Americans, which increases risk of diabetes [16]. A study by Wang et al. [16] etc found brown rice instead of white rice significantly reduced the risk of getting 


\section{Current Research in Diabetes \& Obesity Journal}

diabetes in pre-diabetic Chinese Americans living in New York City.

The study has limitations. Subjects were not recruited from the general community but from a specific primary care clinic for physical checkup or regular follow up. They might be more health conscious and sicker than general population. This primary care clinic was localized in a specific neighborhood in NYC and therefore these results may not be generalizable to the overall Chinese American population. Also, we were unable to compare to insulin glucose tolerance since there were no oral glucose tolerance tests performed, so we could only use FBG as the reference standard to calculate sensitivity of HbA1c. Clearly, more studies need to be done to confirm these findings. Overall, however, $\mathrm{HbA1c}$ is more strongly associated with risks of cardiovascular disease and death from any causes as compared with fasting glucose in addition to risk of diabetes $[17,18]$. Also HbA1c is more accurate that FBG in identifying cases of retinopathy in addition to strong correlation with retinopathy [18]. Therefore HbA1c may be a better predictor of potential medical complications than FBG.

\section{Conclusion}

In a group of Chinese Americans screened in a primary care setting in New York City, HbA1c had much higher sensitivity for diagnosing prediabetes than FBG. Using FBG alone would have significantly under diagnosed prediabetes in this group of patients, which may lead to delayed diagnosis and potential progression to diabetes. Therefore, the best option may be to use $\mathrm{HbA1c}$ as a screening tool in order to get earlier diagnosis of prediabetes. Combining HbA1c with fasting glucose will significantly increase the sensitivity in diagnosing prediabetes in Chinese Americans. This we hope should lead to more cases of prediabetes identified, and early intervention enforced to prevent or delay the progression to diabetes through intensive lifestyle changes, or metformin use, both shown to be equally effective in all ethnic groups $[6,16,19,20]$.

\section{References}

1. (2016) American Diabetes Association. Classification and Diagnosis of Diabetes. Diabetes Care 39(Suppl 1): S13-S22.

2. (2017) National Diabetes Statistics Report, CDC, pp. 1-20.

3. David MN, Mayer BD, Ralph AF, Robert JH, Robert RH, et al. (2007) Impaired fasting glucose and impaired glucose tolerance. Diabetes Care 30(3): 753-759.

4. Zhuo X, Zhang P, Barker L, Albright A, Thompson TJ, et al. (2014) The lifetime cost of diabetes and its implications for diabetes prevention.
Diabetes care 37(9): 2557-2564.

5. http://innovation.cms.gov/initiatives/medicare-diabetespreventionprogram

6. Yeh MC, Heo M, Suchday S, Wong A, Poon E, et al. (2016) Translation of the diabetes prevention program for diabetes risk reduction in chinese immigrants in New York City. Diabet Med 33(4): 547-551.

7. William CH, Maria RGA, Alka MK, Jane LC, Wilfred F (2015) BMI cut points to identify at-risk Asian Americans for type 2 diabetes screening. Diabetes care 38(1):150-158.

8. US Census Bureau, American Community Survey.

9. Mann DM, Carson AP, Shimbo D, Fonseca V, Fox CS, et al. (2010) Impact of HbA1c screening criterion on the diagnosis of pre-diabetes among US adults. Diabetes Care 33(10): 2190-2195.

10. Guo F, Moellering DR, Garvey WT (2014) Use of HbA1c for diagnoses of diabetes and prediabetes: comparison with diagnoses based on fasting and $2 \mathrm{hr}$ glucose values and effects of gender, race, and age. Metab Syndr Relat Disord 12(5): 258-268.

11. Herman WH, Ma Y, Uwaifo G, Haffner S, Kahn SE, et al. (2007) Differences in $\mathrm{A} 1 \mathrm{C}$ by race and ethnicity among patients with impaired glucose tolerance in the diabetes prevention program. Diabetes Care 30(10): 2453-2457.

12. William HH (2016) Are there clinical implication of racial difference in HbA1C? yes, to not consider can do great harm! Diabetes Care 39(8): 1458-1461.

13. Chen P, Takeuchi F, Lee JY, Li H, Wu JY, et al. (2014) Multiple nonglycemic genomic loci are newly associated with Blood level of glycated hemoglobin in East Asians. Diabetes 63(7): 2551-2562.

14. Lear SA, Humphries KH, Kohli S, Chockalingam A, Frohlich JJ, et al. (2007) Visceral adipose tissue accumulation differs according to ethnic background: results of the Multicultural Community Health Assessment Trial (M-CHAT)1,2,3. Am J Clin Nutr 86(2): 353-359.

15. Wander PL, Boyko EJ, Leonetti DL, McNeely MJ, Kahn SE, et al. (2013) Change in visceral adiposity independently predicts a greater risk of developing type 2 diabetes over 10 years in Japanese Americans. Diabetes Care 36(2): 289-293.

16. Bin W, Raj M, Jin X, Weijing C, Xue C, et al. (2013) Effects of a whole rice diet on metabolic parameters and inflammatory markers in prediabetes. e-SPEN 8(1): e15-e20.

17. Elizabeth S, Michael WS, Hong Z, Kunihiro M, Lynne W, et al. (2010) Glycated hemoglobin, diabetes, and cardiovascular risk in nondiabetic adults. N Engl J Med 362(9): 800-811.

18. David KC (2017) Clinical presentation and diagnosis of diabetes mellitus in adults.

19. Sussman JB, Kent DM, Nelson JP, Hayward RA (2015) Improving diabetes prevention with benefit based tailored treatment: risk based reanalysis of diabetes prevention program. BMJ 350: h454.

20. (2015) Diabetes Prevention Program Research Group. HbA1c as a predictor of diabetes and as an outcome in the diabetes prevention program: a randomized clinical trial. Diabetes Care 38(1): 51-58. 
(C) This work is licensed under Creative BY Commons Attribution 4.0 Licens

DOI: 10.19080/CRDOJ.2017.05.555653
Your next submission with Juniper Publishers will reach you the below assets

- Quality Editorial service

- Swift Peer Review

- Reprints availability

- E-prints Service

- Manuscript Podcast for convenient understanding

- Global attainment for your research

- Manuscript accessibility in different formats ( Pdf, E-pub, Full Text, Audio)

- Unceasing customer service

Track the below URL for one-step submission https://juniperpublishers.com/online-submission.php 